\title{
Hidroquímica y su variabilidad espacio temporal en un bofedal altoandino de la Reserva Paisajística Nor Yauyos Cochas, Perú
}

\author{
Hydrochemistry and its temporal space variability in a high-altitude bofedal of the \\ Nor Yauyos Cochas Landscape Reserve, Peru
}

\author{
Alfredo Manho Choy Galindo ${ }^{1}$, Mario Antonio Anaya Raymundo ${ }^{*}$
}

\begin{abstract}
RESUMEN
La Reserva Paisajística Nor Yauyos Cochas (RPNYC) presenta bofedales altoandinos, en cuyas turberas se encuentran contenidas sus aguas subterráneas, las cuales están influenciadas por su hidroquímica. Así, estos ecosistemas son importantes fuentes de alimentación, agua, refugio y lugar de anidación para una diversidad de aves y animales silvestres. El objetivo de la investigación fue generar conocimiento sobre la química del agua depositada en el bofedal del sector Moyobamba, a través del proceso de caracterización hidroquímica y su variabilidad espacio temporal. La metodología se basó en el registro de las propiedades fisicoquímicas (pH, temperatura y CE) de 34 pozos distribuidos estratégicamente en toda el área del bofedal, y el análisis de constituyentes mayoritarios correspondiente a una muestra de 10 pozos (aniones: $\mathrm{HCO}_{3}{ }^{-}, \mathrm{SO}_{4}{ }^{2-}, \mathrm{Cl}^{-}, \mathrm{CO}_{3}{ }^{-2} \mathrm{y}$ cationes: $\mathrm{Na}^{+}, \mathrm{K}^{+} ; \mathrm{Ca}^{+2} ; \mathrm{Mg}^{+2}$ ). Las mediciones se realizaron considerando el agrupamiento en tres sectores y por temporada (mayo, julio y octubre del 2017, temporada seca y febrero del 2018, temporada húmeda). Los datos fueron analizados a partir del diagrama de Piper (\% meq/l) y pruebas estadísticas específicas (Descriptivos, ANOVA y correlación). El tipo de agua dominante fue la bicarbonatada cálcica $\left(\mathrm{HCO}_{3}{ }^{-} \mathrm{Ca}^{+2}\right)$ en el $95 \%$ de los pozos y la bicarbonatada magnésica $\left(\mathrm{HCO}_{3}{ }^{-}-\mathrm{Mg}^{+2}\right)$ en el 5\% restante, influenciadas por las calizas presentes en sus formaciones geológicas. Los iones $\mathrm{HCO}_{3}{ }^{-}, \mathrm{Ca}^{+2}, \mathrm{Mg}^{+2}$ y $\mathrm{Na}^{+}$registraron un aumento en su concentración y CE a medida que transcurría la temporada seca. Mientras que el pH del agua presentó un carácter ligeramente ácido $(\mathrm{pH} \cong 6,6)$, con temperatura promedio de $8,1^{\circ} \mathbf{C}$, siendo considerado este valor normal para este tipo de humedales. Se encontró correlación positiva media entre los iones bicarbonato $\left(\mathrm{HCO}_{3}^{-}\right)$y calcio $\left(\mathrm{Ca}^{+2}\right)$, y una correlación positiva débil entre el $\mathrm{pH}$ y la $\mathrm{CE}$.

Palabras clave: bofedal, Andes, humedal, hidroquímica, agua subterránea
\end{abstract}

\begin{abstract}
The Nor Yauyos Cochas Landscape Reserve (RPNYC) presents high Andean wetlands, whose peat bogs contain their subterranean waters, which are influenced by their hydrochemistry. Thus, these ecosystems are important sources of food, water, shelter and nesting place for a diversity of birds and wild animals. The objective of the research was to generate knowledge about the chemistry of the water deposited in the wetland of the Moyobamba sector, through the process of hydrochemical characterization and its temporal space variability. The methodology was based on the recording of the physicochemical properties ( $\mathrm{pH}$, temperature and $\mathrm{EC})$ of 34 wells strategically distributed throughout the bofedal area, and the majority constituent analysis corresponding to a sample of 10 wells (anions: $\mathrm{HCO}_{3}{ }^{-} \mathrm{SO}_{4}^{2}, \mathrm{Cl}, \mathrm{CO}_{3}^{-2}$ and cations: $\mathrm{Na}^{+}, \mathrm{K}^{+}, \mathrm{Ca}^{+2}$, $\mathrm{Mg}^{+2}$ ). The measurements were made considering the grouping in three sectors and by season (May, July and October of 2017, dry season and February of 2018, wet season). The data were analyzed from the Piper diagram (\% meq/l) and specific statistical tests (Descriptive, ANOVA and correlation). The dominant water type was calcium bicarbonated $\left(\mathrm{HCO}_{3}^{-}-\mathrm{Ca}^{+2}\right)$ in $95 \%$ of the wells and magnesium bicarbonated $\left(\mathrm{HCO}_{3}^{-}-\mathrm{Mg}\right.$ ${ }^{+2}$ ) in the remaining 5\%, influenced by the limestone present in their formations geological the $\mathrm{HCO}_{3}, \mathrm{Ca}^{+2}, \mathrm{Mg}^{+2}$ and $\mathrm{Na}^{+}$ions registered an increase in their concentration and EC as the dry season passed. While the $p H$ of the water presented a slightly acid character $(p H \cong 6,6)$, with an average temperature of $8,1^{\circ} \mathrm{C}$; this normal value is considered for this type of wetlands. A positive mean correlation was found between bicarbonate ions $\left(\mathrm{HCO}_{3}{ }^{-}\right)$and calcium $\left(\mathrm{Ca}^{+2}\right)$ and a weak positive correlation between $\mathrm{pH}$ and $\mathrm{EC}$.
\end{abstract}

Key words: bofedal, Andes, wetland, hydrochemistry, groundwater

\section{Introducción}

La Reserva Paisajística Nor Yauyos Cochas (RPNYC) es un área natural protegida, ubicada entre los departamentos de Lima y Junín (Perú), que se caracteriza por albergar varios bofedales altoandinos. La importancia que tienen estos ecosistemas dentro de la reserva se debe a que constituyen fuentes de

1 Facultad de Ingeniería Agraria. Universidad Católica Sedes Sapientiae. Esq. Constelaciones y Sol de Oro s/n Urb. Sol de Oro. Los Olivos, Lima (Perú).

* Autor para correspondencia: manaya@ucss.edu.pe

Fecha de recepción: 06 enero, 2018.

Fecha de aceptación: 10 septiembre, 2018.

DOI: 
alimentación, agua, refugio y sitios de anidación para varios animales domésticos y silvestres (Maldonado, 2015; Salvador et al., 2015; Korswagen, 2016). Asimismo, los bofedales son ecosistemas muy sensibles al cambio climático, siendo su turba la característica más vulnerable. Es por ello que, para protegerlos, existe la necesidad de estudiar su hidroquímica (Charman, 2009; Squeo et al., 2006). Las turbas de los bofedales pueden clasificarse de acuerdo a su origen hidroquímico: las que reciben aguas de fuentes atmosféricas, ombrotróficas; y las que reciben de escorrentía superficial y aguas subterráneas (contienen minerales del suelo), minerotróficas (Charman, 2009).

En los bofedales altoandinos, las aguas subterráneas que se encuentran contenidas dentro de sus restos vegetales que conforman su turba presentan una geoquímica que influye sobre su tipo de vegetación (Cooper et al., 2010).

La justificación del trabajo se basa en los escasos estudios sobre las características químicas de las aguas subterráneas de los bofedales altoandinos. Solo se ha estudiado ampliamente sus comunidades vegetales y su flora (Ruthsatz et al., 2012). De esta manera, se busca brindar información que sirva como línea base para el desarrollo de futuras investigaciones y la toma de decisiones en la gestión de estas áreas.

En la presente investigación se realizó un estudio de las aguas subterráneas contenidas en la turba de los bofedales altoandinos de la RPNYC, sector Moyobamba, a través del proceso de caracterización hidroquímica y su variabilidad espacio temporal. Específicamente, los objetivos del estudio fueron: 1) determinar los tipos de agua dominantes en el bofedal de Moyobamba; 2) realizar la caracterización espacio temporal de sus constituyentes mayoritarios; 3 ) realizar el análisis espacio temporal de sus propiedades fisicoquímicas; 4) realizar un análisis correlacional entre las variables medidas.

\section{Materiales y métodos}

\section{Área de estudio}

El estudio se realizó en el bofedal altoandino del sector Moyobamba, que se ubica en la cuenca del río Cochas Pachacayo, sector norte de la RPNYC. El bofedal pertenece al distrito de Canchayllo, provincia de Jauja (Región de Junín, Perú) (ver Figura 1). Se

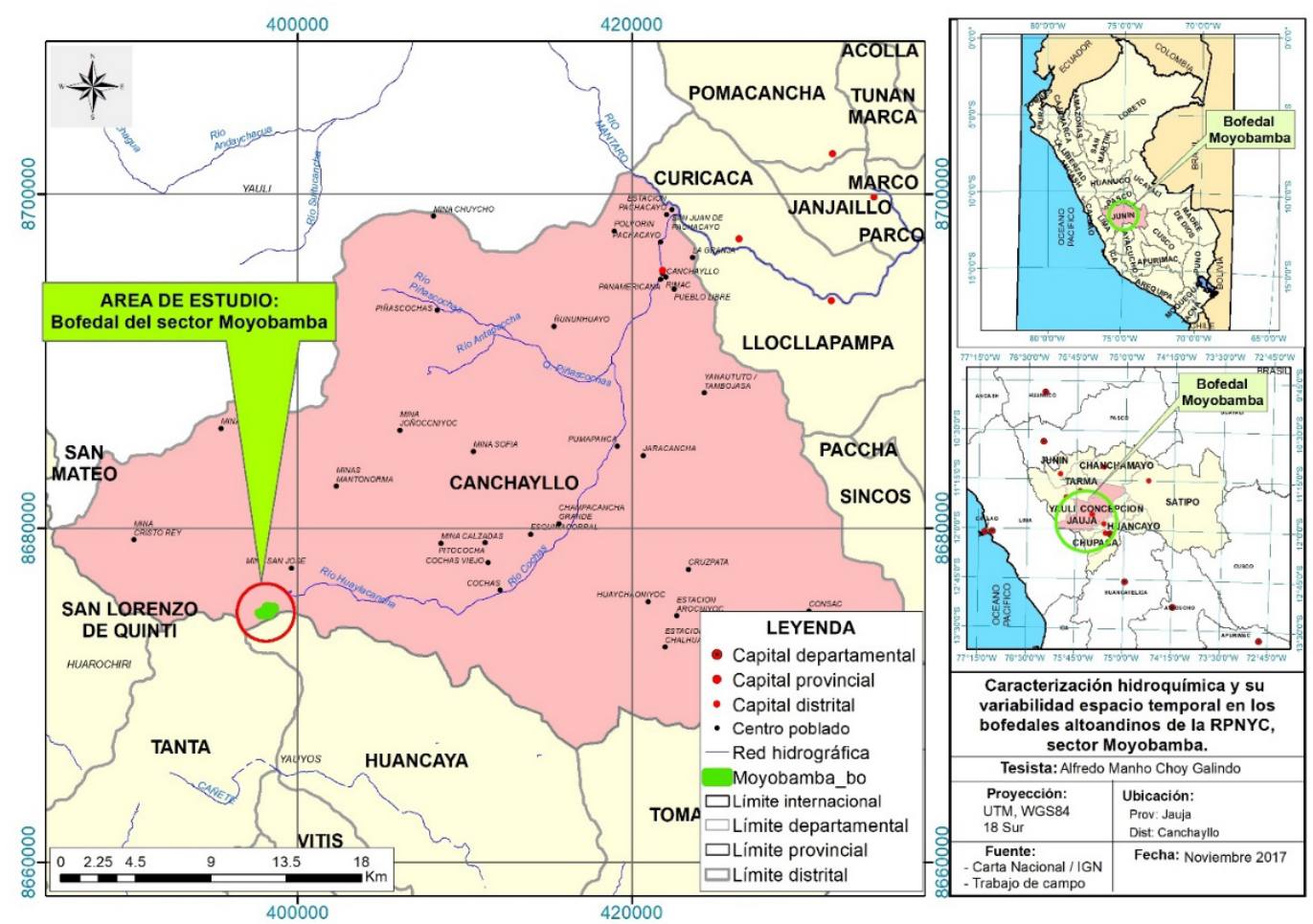

Figura 1. Mapa de localización del bofedal de Moyobamba. 
ubica entre los 4.600 y 4.700 msnm, y abarca una extensión de $\sim 1 \mathrm{~km}$ y una superficie de $\sim 0,6 \mathrm{~km}^{2}$. Su territorio pertenece a la Comunidad Campesina de Tanta (Cañete - Lima), la cual está siendo conservada y protegida para el uso exclusivo como hábitat para vicuñas (Korswagen, 2016).

El bofedal de Moyobamba presenta dos temporadas marcadas: la húmeda, entre noviembre y abril, con clima templado y lluvias constantes; y la seca, entre mayo y octubre con escasas lluvias y altas temperaturas durante el día, que descienden bajo cero en las noches generando las heladas (Oyague y Maldonado, 2015; Salvador et al., 2015). Sus terrenos se encuentran atravesados por canales de drenaje y cauces de deslizamientos, que lo dividen en tres sectores, y que se alimentan probablemente tanto de aguas subterráneas como de aguas superficiales (Durán et al., 2009). Según Holden et al., (2004), la instalación de zanjas de drenaje en la turba podría tener un impacto en los componentes químicos del agua.

\section{Monitoreo de pozos y estudios hidroquímicos}

En el bofedal de Moyobamba se instalaron 34 pozos de monitoreo, utilizados para el registro de las propiedades fisicoquímicas ( $\mathrm{pH}, \mathrm{CE}$ y temperatura). De estos, 10 fueron seleccionados para la toma de muestras y el análisis correspondiente de los constituyentes mayoritarios $\left(\mathrm{Ca}^{+2}, \mathrm{Cl}^{-}, \mathrm{Na}^{+}, \mathrm{K}^{+}\right.$, $\mathrm{HCO}_{3}^{-}, \mathrm{CO}_{3}^{-2}, \mathrm{SO}_{4}^{-2}$ ) (ver Figura 2). El monitoreo de los pozos se ejecutó cada dos meses: tres veces durante la temporada seca comprendida entre los meses de mayo y octubre del 2017 y una durante la temporada húmeda, en el mes de febrero del 2018 (Flusche et al., 2005).

La metodología para distribuir los pozos de monitoreo se basó fundamentalmente en tres criterios: la presencia significativa de turba, la cercanía con los canales de drenaje y la formación de una cuadrícula (con líneas paralelas), para obtener una distribución más homogénea de los pozos en el bofedal (ver Figura 3). La instalación de los pozos de monitoreo en el bofedal se inició con la ubicación del punto, siguiendo los criterios anteriores. A continuación, se realiza la perforación del suelo de la turba, hasta alcanzar 1,5 metros de profundidad máxima, usando un barreno metálico circular. Finalmente, el hoyo se refuerza introduciéndole un tubo de PVC, de 1,5 metros de longitud máxima, dos pulgadas de diámetro y con perforaciones.

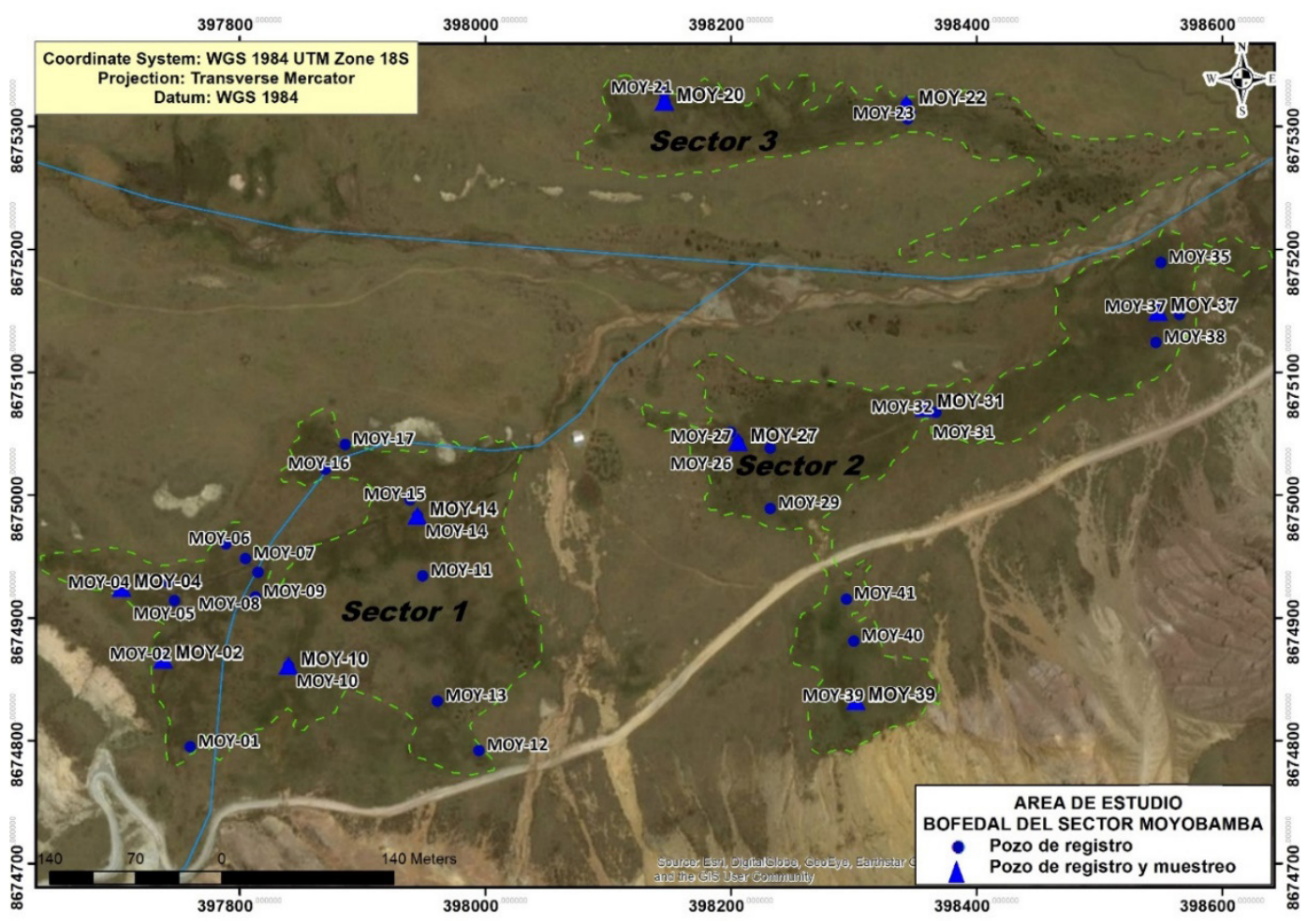

Figura 2. Pozos de monitoreo distribuidos en el bofedal de Moyobamba. 


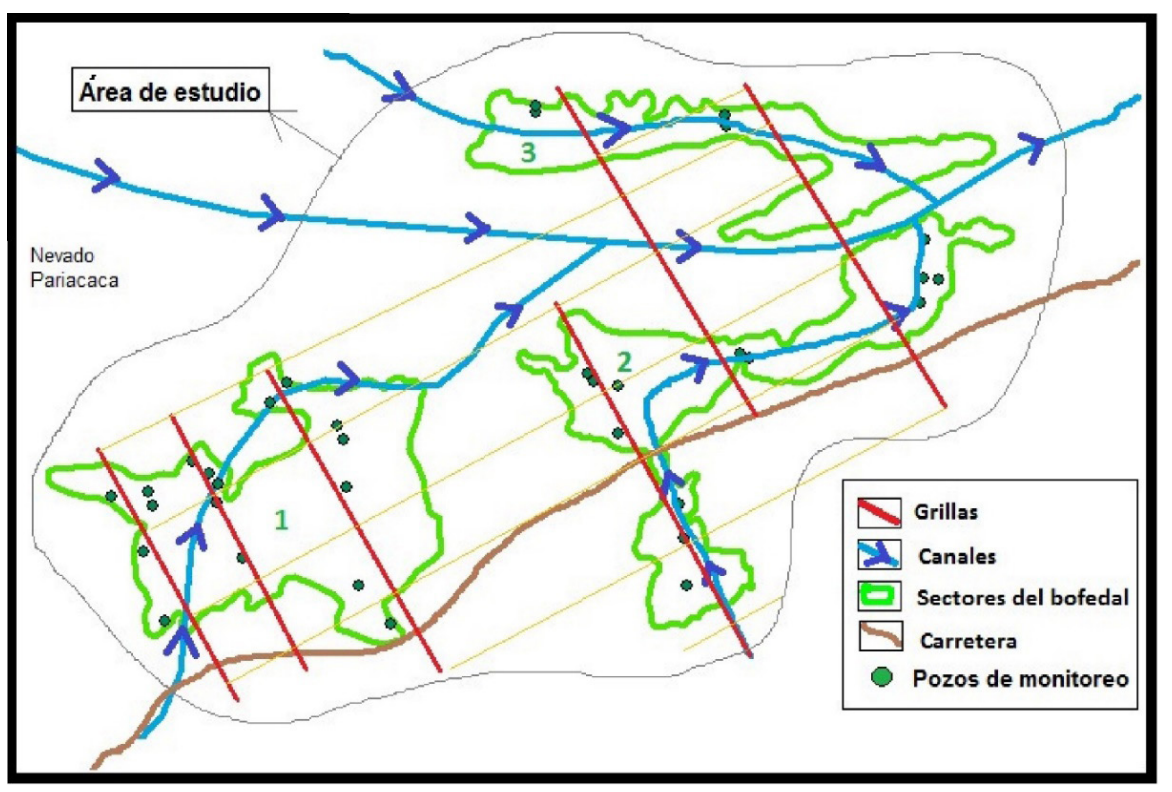

Figura 3. Diseño de distribución de los pozos de monitoreo, bofedal de Moyobamba.

Asimismo, se realizó la purga de los pozos utilizando un tubo hueco (bailer), con el que se extrajo el sedimento, hasta que el agua obtenida fuera idónea para el muestreo. Se buscó obtener el mayor volumen de muestras de agua del pozo y una mayor representatividad de la altura de la turba. Para la adecuada conservación y ubicación, se procede a colocar una tapa al pozo, la cual se rotula y se le agrega un recinto, y se georreferencian sus coordenadas (Romanelli et al., 2010).

Para el registro de las propiedades fisicoquímicas se utilizó un equipo multiparámetro de marca HANNA HI9813-6, previamente calibrado en campo. Mientras que para la recolección de muestras se bombeó manualmente agua del pozo y se utilizaron botellas de polietileno de un litro de capacidad, las cuales fueron previamente enjuagadas con agua del pozo y refrigeradas para su análisis en el laboratorio de Agua, Suelo, Medio Ambiente y Fertirriego
(LAASMA - UNALM). Se consideraron las normas para agua potable y residual APHA, AWWA, WPCF 17th Ed.1992 (Cooper et al., 2010; Romanelli et al., 2010; Vidal et al., 2011) (ver Tabla 1).

Los datos fueron analizados considerando métodos no estadísticos, como el diagrama de Piper (meq/l), que permite conocer el tipo de agua en los pozos, utilizando el programa AQUACHEM 4.0 (Romanelli et al., 2010; Sánchez et al., 2015). Asimismo, se aplicaron métodos estadísticos ( $\mathrm{mg} / \mathrm{l}$ ) para evaluar el comportamiento espacio temporal de las variables fisicoquímicas y su correlación, utilizando el programa IBM SPSS Statistics versión 20 (Estadísticos descriptivos, análisis de varianza [ANOVA], correlación de Pearson y de Spearman) (Ginocchio et al., 2008).

Por otra parte, se localizó el bofedal de Moyobamba y se identificó su geología utilizando el software ArcGis 10.5 (ver Figura 4).

Tabla 1. Métodos empleados para el análisis de los constituyentes mayoritarios

\begin{tabular}{cccc}
\hline Aniones & APHA, AWWA, WPCF 17 th Ed. 1992. & Cationes & APHA, AWWA, WPCF 17 ${ }^{\text {th }}$ Ed. 1992. \\
\hline $\mathrm{HCO}_{3^{-}}$ & Método titulométrico & $\mathrm{Na}^{+}$ & Método fotométrico de emisión de llama \\
$\mathrm{CO}_{3}^{-2}$ & Método de titulación & $\mathrm{Ca}^{+2}$ & Método espectrométrico \\
$\mathrm{Cl}^{-}$ & Método argentométrico & $\mathrm{K}^{+}$ & de absorción atómica \\
$\mathrm{SO}_{4}^{-2}$ & Método turbidimétrico & $\mathrm{Mg}^{+2}$ & de llama \\
\hline Fuente: Laboratorio de agua, suelo, medio ambiente y fertirriego. UNALM - FIA (APHA, AWWA y WPCF, 1992).
\end{tabular}




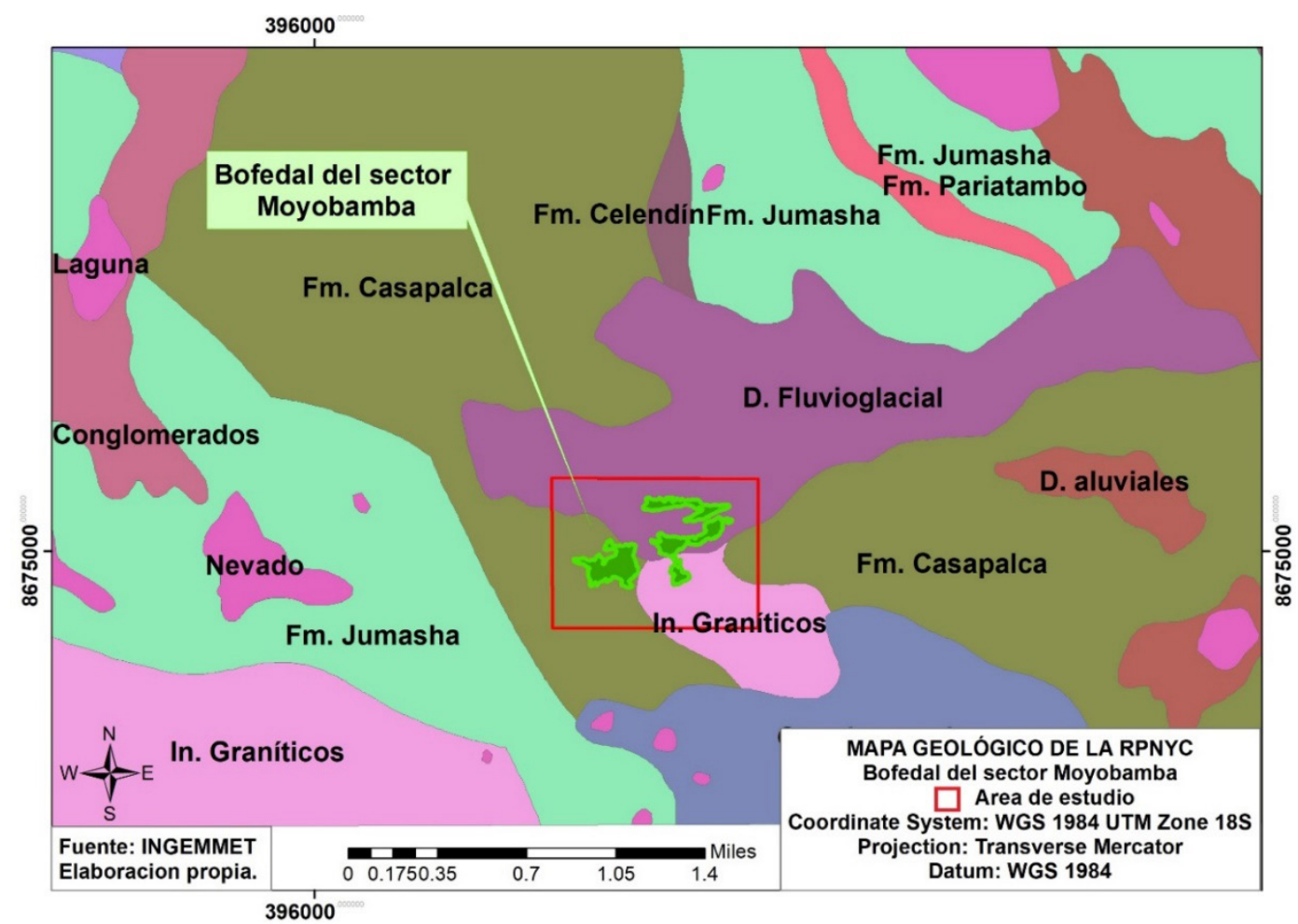

Figura 4. Mapa geológico del bofedal de Moyobamba.

\section{Resultados y discusión}

\section{Resultados}

Los resultados presentan como tipo de agua dominante la bicarbonatada cálcica, que representa el $95 \%$ de las muestras del total, mientras que el 5\% restante lo representó el tipo de agua bicarbonatada magnésica. En la Figura5, se observa la caracterización hidroquímica de los muestreos realizados. En primer lugar, en el triángulo de cationes, todos los pozos tienden a agruparse en la zona de tipo cálcico, siendo el pozo MOY 39 el único que se encuentra separado. En segundo lugar, en el triángulo de aniones, todos los pozos tienden a agruparse en la zona de tipo bicarbonatado, siendo el pozo MOY 31 el único que se encuentra separado del grupo y ubicado en la zona de tipo bicarbonatado sulfatado. Por último, en el rombo se observa que todos los pozos de monitoreo se encuentran agrupados en la zona de tipo bicarbonatadas cálcicas y/o magnésicas, siendo el pozo MOY 31 el que está separado del grupo y al límite de la zona.

En la Tabla 2, se observa el promedio por sector y general de los constituyentes mayoritarios del muestreo realizado en los pozos del bofedal del sector Moyobamba. La secuencia catiónica promedio (mg/l) fue: $\mathrm{Ca}^{+2}(71,30)>\mathrm{Mg}^{+2}(14,22)>\mathrm{Na}^{+}(4,44)$ $>\mathrm{K}^{+}(1,69)$, mientras que la aniónica fue de $\mathrm{HCO}_{3}{ }^{-}$ $(210,45)>\mathrm{SO}_{2}^{-2}(42,24)>\mathrm{Cl}^{-}(22,69)>\mathrm{CO}_{3}^{-2}(0)$.

Tabla 2. Promedio sectorial y general para los constituyentes mayoritarios (mg/l), bofedal de Moyobamba.

\begin{tabular}{lrrrr}
\hline IONES & Sector 1 & Sector 2 & Sector 3 & General \\
\hline $\mathrm{HCO}_{3}{ }^{-}$ & 209,24 & 198,80 & 236,15 & 210,45 \\
$\mathrm{SO}_{4}^{2-}$ & 46,16 & 46,77 & 25,31 & 42,24 \\
$\mathrm{Cl}^{-}$ & 24,15 & 19,58 & 26,00 & 22,69 \\
$\mathrm{Ca}^{2+}$ & 71,63 & 67,87 & 77,48 & 71,30 \\
$\mathrm{Mg}^{2+}$ & 15,43 & 14,03 & 12,18 & 14,22 \\
$\mathrm{~K}^{+}$ & 2,46 & 1,22 & 1,07 & 1,69 \\
$\mathrm{Na}^{+}$ & 4,39 & 4,12 & 5,17 & 4,44 \\
\hline
\end{tabular}

Por medio del ANOVA, se obtuvo que el potasio presentó una variación significativa entre el sector 1 $(\mathrm{p}=0,007<0,05)$ y los demás sectores (ver Tabla 3 ).

Los estadísticos descriptivos demostraron que existió un aumento en la concentración del anión bicarbonato y los cationes $\mathrm{Ca}^{+2}, \mathrm{Mg}^{+2}$ y Na${ }^{+}$, a medida que transcurría la temporada seca de mayo hasta octubre, con promedios mínimos y máximos (mg/l) 
DIAGRAMA GENERAL

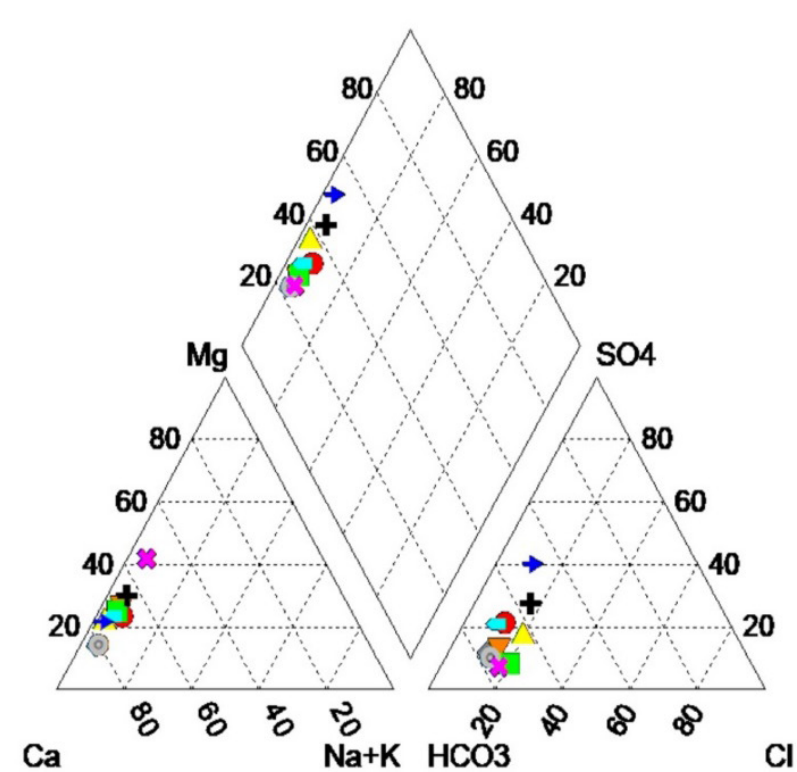

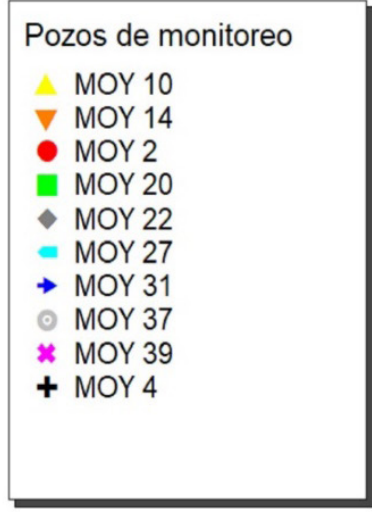

Figura 5. Diagrama de Piper a nivel general ( $\%$ meq/l).

Tabla 3. Prueba de Tukey por sector del ión $\mathrm{K}^{+}$

\begin{tabular}{llc}
\hline (I) Sector del bofedal & (J) Sector del bofedal & Sig. \\
\hline \multirow{2}{*}{ Sector 1 } & Sector 2 & 0,010 \\
& Sector 3 & 0,021 \\
\hline \multirow{2}{*}{ Sector 2 } & Sector 1 & 0,010 \\
& Sector 3 & 0,954 \\
\hline \multirow{2}{*}{ Sector 3 } & Sector 1 & 0,021 \\
& Sector 2 & 0,954 \\
\hline
\end{tabular}

de (184,2 - 226), (60,5 - 80,7), (13,4 - 15,7), (2,2 9,2), respectivamente (ver Figuras del $6-9$ ).

Por medio del ANOVA, se obtuvo que el cloruro $(p=0<0,05)$ y el sodio $(p=0<0,05)$, registraron una variación significativa entre octubre y los demás meses, mientras que el potasio $(p=0,002<0,05)$, entre julio y los meses de octubre y febrero (ver Tabla 4 ).

La conductividad eléctrica promedio de las aguas subterráneas del bofedal de Moyobamba fue de $373,4 \mu \mathrm{S} / \mathrm{cm}$. El pH registró un valor promedio, con carácter ligeramente ácido $(\mathrm{pH} \cong 6,6)$, mientras que la temperatura promedio del agua fue de $8,1^{\circ} \mathbf{C}$, alcanzando un mínimo de 7,9 en el sector 1 y un máximo de 8,6 en el sector 3 (ver Tabla 5).

Por otra parte, se comprobó un incremento de la conductividad eléctrica $(\mu \mathrm{S} / \mathrm{cm})$ a partir del mes de mayo $(391,4)$ hasta julio (temporada seca), donde alcanza su máximo valor $(523,2)$. Luego, la CE descendió hasta alcanzar su menor valor $(263,8)$, en el mes de febrero (temporada húmeda) (ver Figura 10). Igualmente, el $\mathrm{pH}$ obtuvo valores promedios ligeramente alcalinos en los meses de mayo $(\mathrm{pH}=$ $7,1)$ y julio $(\mathrm{pH}=7,2)$, y ligeramente ácidos en los meses de octubre $(\mathrm{pH}=5,9)$ y febrero $(\mathrm{pH}=6,3)$ (ver Figura 11).

A nivel general, en el bofedal de Moyobamba, con relación a los constituyentes mayoritarios, se encontró una correlación positiva media entre los iones bicarbonato $\left(\mathrm{HCO}_{3}^{-}\right)$y calcio $\left(\mathrm{Ca}^{+2}\right)(\mathrm{r}=0,656)$ (ver Tabla 6); y en el mes de febrero, una correlación positiva media entre los iones bicarbonato $\left(\mathrm{HCO}_{3}^{-}\right)$y magnesio $\left(\mathrm{Mg}^{+2}\right)(\mathrm{r}=0,678)$ (ver Tabla 7). Por otro lado, también se observó una correlación significativa positiva débil $(r=0,487)$ entre el $\mathrm{pH}$ y la $\mathrm{CE}$.

\section{Discusiones}

Según Cooper et al. (2010), la geología de un bofedal influye sobre la composición química de las aguas subterráneas contenidas en su turba. Así, los resultados obtenidos en la presente investigación, con respecto a los constituyentes mayoritarios del bofedal de Moyobamba, son similares a los registrados en su 


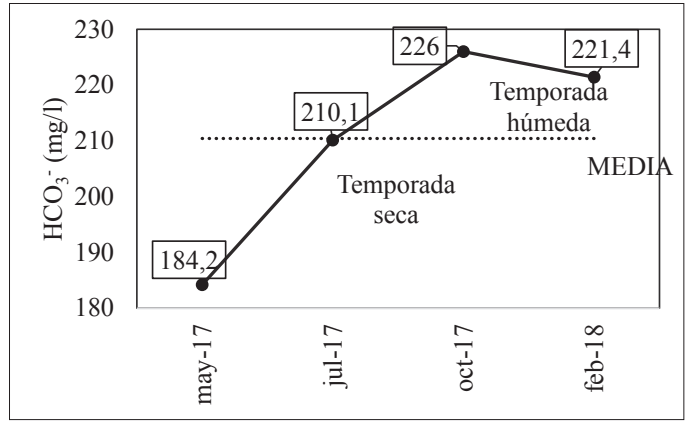

Figura 6. Variación temporal del bicarbonato.

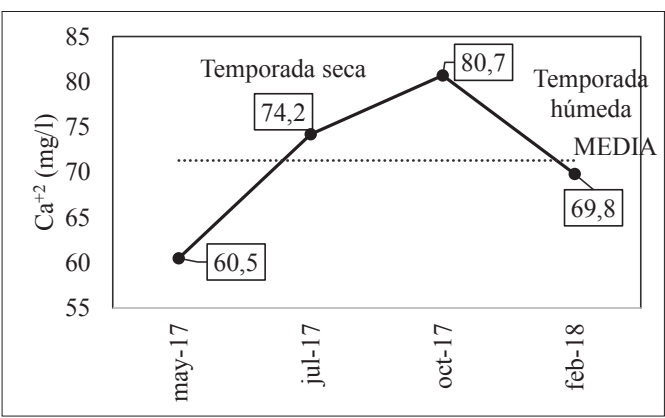

Figura 7. Variación temporal del calcio.

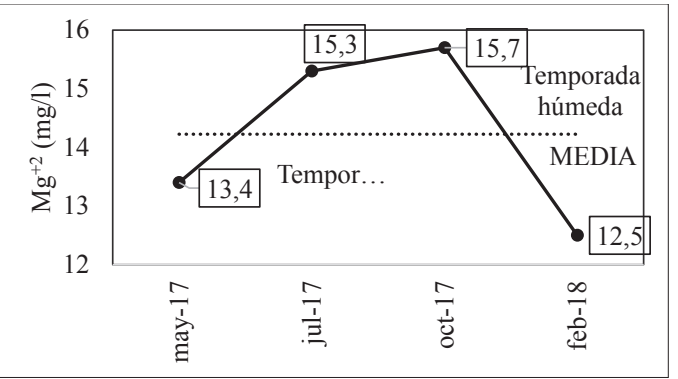

Figura 8. Variación temporal del magnesio.

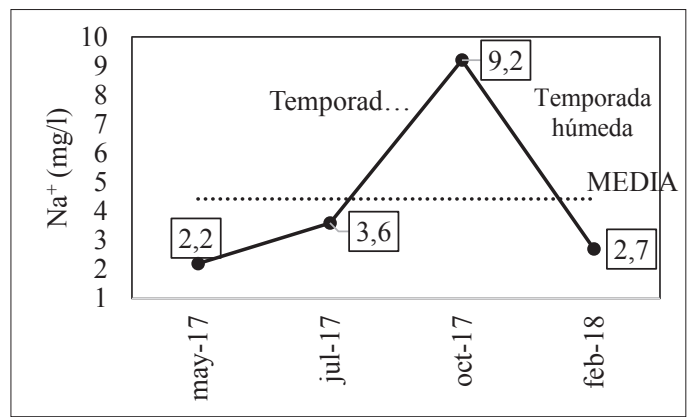

Figura 9. Variación temporal del sodio.
Tabla 4. Prueba de Tukey por mes

\begin{tabular}{|c|c|c|c|}
\hline $\begin{array}{c}\text { Variable } \\
\text { dependiente }\end{array}$ & $\begin{array}{r}\text { Temporada } \\
\text { de muestreo }\end{array}$ & $\begin{array}{r}\text { Temporada } \\
\text { de muestreo }\end{array}$ & Sig. \\
\hline \multirow{12}{*}{$\mathrm{Cl}^{-}$} & \multirow{3}{*}{ MAYO } & JULIO & 0,892 \\
\hline & & OCTUBRE & 0,006 \\
\hline & & FEBRERO & 0,103 \\
\hline & \multirow{3}{*}{ JULIO } & MAYO & 0,892 \\
\hline & & OCTUBRE & 0,001 \\
\hline & & FEBRERO & 0,365 \\
\hline & \multirow{3}{*}{ OCTUBRE } & MAYO & 0,006 \\
\hline & & JULIO & 0,001 \\
\hline & & FEBRERO & 0,000 \\
\hline & \multirow{3}{*}{ FEBRERO } & MAYO & 0,103 \\
\hline & & JULIO & 0,365 \\
\hline & & OCTUBRE & 0,000 \\
\hline \multirow{12}{*}{$\mathrm{Na}^{+}$} & \multirow{3}{*}{ MAYO } & JULIO & 0,163 \\
\hline & & OCTUBRE & 0,000 \\
\hline & & FEBRERO & 0,857 \\
\hline & \multirow{3}{*}{ JULIO } & MAYO & 0,163 \\
\hline & & OCTUBRE & 0,000 \\
\hline & & FEBRERO & 0,546 \\
\hline & \multirow{3}{*}{ OCTUBRE } & MAYO & 0,000 \\
\hline & & JULIO & 0,000 \\
\hline & & FEBRERO & 0,000 \\
\hline & \multirow{3}{*}{ FEBRERO } & MAYO & 0,857 \\
\hline & & JULIO & 0,546 \\
\hline & & OCTUBRE & 0,000 \\
\hline \multirow{12}{*}{$\mathrm{K}^{+}$} & \multirow{3}{*}{ MAYO } & JULIO & 0,094 \\
\hline & & OCTUBRE & 0,530 \\
\hline & & FEBRERO & 0,603 \\
\hline & \multirow{3}{*}{ JULIO } & MAYO & 0,094 \\
\hline & & OCTUBRE & 0,003 \\
\hline & & FEBRERO & 0,004 \\
\hline & \multirow{3}{*}{ OCTUBRE } & MAYO & 0,530 \\
\hline & & JULIO & 0,003 \\
\hline & & FEBRERO & 0,999 \\
\hline & \multirow{3}{*}{ FEBRERO } & MAYO & 0,603 \\
\hline & & JULIO & 0,004 \\
\hline & & OCTUBRE & 0,999 \\
\hline
\end{tabular}

Tabla 5. Promedio sectorial y general para las propiedades fisicoquímicas, bofedal de Moyobamba.

\begin{tabular}{ccccc}
\hline & Sector 1 & Sector 2 & Sector 3 & General \\
\hline $\mathrm{CE}(\mu \mathrm{S} / \mathrm{cm})$ & 382,3 & 374,1 & 333,7 & 373,4 \\
$\mathrm{pH}$ & 6,6 & 6,7 & 6,5 & 6,6 \\
$\mathrm{~T}\left({ }^{\circ} \mathrm{C}\right)$ & 7,9 & 8,4 & 8,6 & 8,1
\end{tabular}




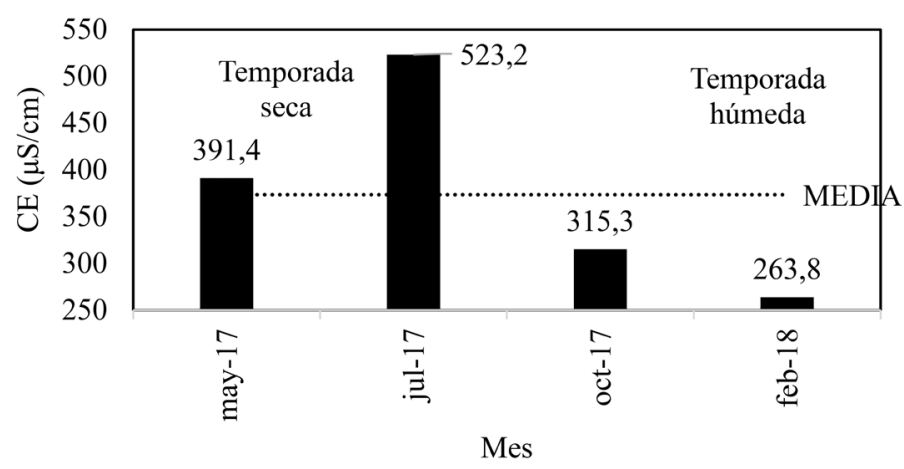

Figura 10. Variación temporal de la conductividad eléctrica.

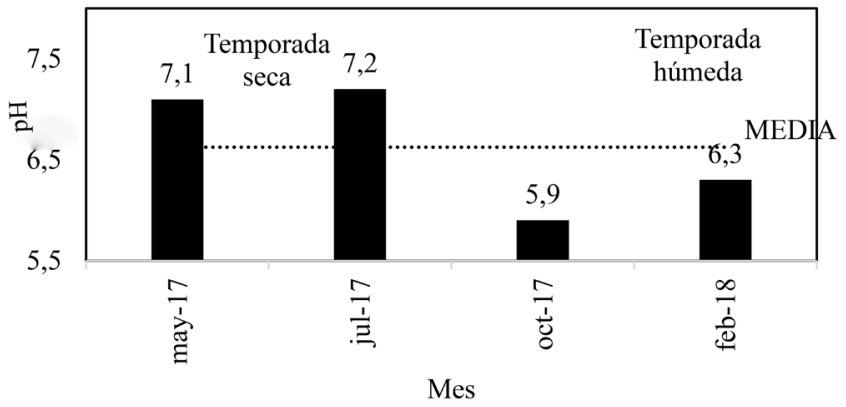

Figura 11. Variación temporal del pH.

Tabla 6. Correlación de Pearson a nivel general

\begin{tabular}{ccccc}
\hline & & $\mathbf{H C O}_{3}{ }^{-}$ & $\mathbf{C a}^{2+}$ & $\mathbf{M g}^{2+}$ \\
\hline \multirow{3}{*}{$\mathbf{H C O}_{3^{-}}$} & Correlación de Pearson & 1 & $0,656^{* *}$ & 0,166 \\
& Sig. (bilateral) & & 0,000 & 0,307 \\
& $\mathrm{~N}$ & 40 & 40 & 40 \\
\cline { 2 - 5 } $\mathbf{C a}^{2+}$ & Correlación de Pearson & $0,656^{* *}$ & 1 & $-0,084$ \\
& Sig. (bilateral) & 0,000 & & 0,608 \\
& $\mathrm{~N}$ & 40 & 40 & 40 \\
\cline { 2 - 5 } $\mathbf{M g}^{2+}$ & Correlación de Pearson & 0,166 & $-0,084$ & 1 \\
& Sig. (bilateral) & 0,307 & 0,608 & \\
& $\mathrm{~N}$ & 40 & 40 & 40
\end{tabular}

** La correlación es significativa al nivel 0,01 .
Tabla 7. Correlación de Pearson para el mes de febrero

\begin{tabular}{|c|c|c|c|c|}
\hline & & $\mathrm{HCO}_{3}^{-}$ & $\mathrm{Ca}^{2+}$ & $\mathbf{M g}^{2+}$ \\
\hline & Correlación de Pearson & 1 & $0,864^{* *}$ & $0,678^{*}$ \\
\hline \multirow[t]{3}{*}{$\mathrm{HCO}_{3}^{-}$} & Sig. (bilateral) & & 0,001 & 0,031 \\
\hline & $\mathrm{N}$ & 10 & 10 & 10 \\
\hline & Correlación de Pearson & $0,864^{* *}$ & 1 & 0,377 \\
\hline \multirow[t]{3}{*}{$\mathrm{Ca}^{2+}$} & Sig. (bilateral) & 0,001 & & 0,283 \\
\hline & $\mathrm{N}$ & 10 & 10 & 10 \\
\hline & Correlación de Pearson & $0,678^{*}$ & 0,377 & 1 \\
\hline \multirow[t]{2}{*}{$\mathbf{M g}^{2+}$} & Sig. (bilateral) & 0,031 & 0,283 & \\
\hline & $\mathrm{N}$ & 10 & 10 & 10 \\
\hline
\end{tabular}

* La correlación es significativa al nivel 0,05 .

** La correlación es significativa al nivel 0,01 . 
estudio, Turberas alpinas de los Andes (región Jalca - Cajamarca), donde a través del diagrama de Piper, demostró que uno de los tipos de agua dominantes era la bicarbonatada cálcica, Estos resultados se obtuvieron de muestras que fueron recolectadas durante la estación seca y que eran influenciadas por la presencia de rocas carbonatadas (calizas). Asimismo, en el estudio realizado en la vega andina de Tambo - Puquios (Región de Coquimbo - Chile), se observó que las características químicas de sus aguas estuvieron relacionadas con el sustrato geológico de sus montañas y su elevada variabilidad temporal, con el patrón pluviométrico del lugar (Cepeda, 2013; Cepeda \& Armijo, 2014).

Se debe tener en cuenta que durante la temporada seca, los sectores del bofedal de Moyobamba dependen principalmente de sus aguas subterráneas, cuya geoquímica se encuentra influenciada por la formación Jumasha y Casapalca, la cual presenta rocas calizas (dolomita y calcita), compuestas de carbonato de calcio y/o magnesio (MINAM, 2011).

Según Lecomte et al. (2011), la concentración de los constituyentes mayoritarios es influenciada por la variación estacional, aumentando su concentración en la temporada seca y disminuyendo en la temporada húmeda. En efecto, a medida que transcurre la temporada seca, la ausencia de lluvias y el bajo volumen de agua coinciden con el aumento de la concentración del bicarbonato y demás cationes. En esta temporada, el bofedal de Moyobamba depende principalmente de aguas subterráneas de origen carbonatado. Asimismo, en el mes de febrero, temporada húmeda, la concentración de los cationes y aniones mencionados anteriormente disminuyó su valor. En esta temporada existe mayor presencia de lluvias que aportan un mayor volumen de agua.

Las variaciones significativas en la concentración de los iones potasio, sodio y cloruro, entre octubre y los demás meses, se deben probablemente, al inicio de las primeras lluvias, que limpian la turba y el terreno. Mientras que la variación significativa de la concentracion del ion potasio, entre el sector 1 y los demás sectores, se debe probablemente a la diferencia entre las formaciones geológicas de estos (Megard et al., 1996).

Respecto a los resultados de CE obtenidos, son similares a los mencionados por Oyague y Maldonado (2015), donde sus registros más elevados los alcanzó en la temporada seca.

Con relación al carácter ligeramente ácido de las aguas del bofedal de Moyobamba, según Cooper et al. (2010), en particular, las aguas subterráneas provenientes de piedras calizas, tenderán a producir aguas alcalinas y si provienen de piedras con presencia de compuestos sulfatados, producirán aguas ácidas. Sin embargo, esto también puede deberse al proceso de descomposición que sucede en la turba del bofedal, el cual libera sustancias ácidas con carácter ácido que hacen que el pH disminuya (Charman, 2009; Vásquez et al., 2006). En efecto, la descomposicion que ocurre en la parte inferior de la turba (catotelmo), que se encuentra permanentemente saturada de agua a diferencia de la superior (acrotelmo), genera que el agua adquiera un carácter ligeramente ácido (Belyea \& Malmer, 2004).

Los valores de temperatura $\left(\mathrm{T}=8,1^{\circ} \mathrm{C}\right)$ son similares a los obtenidos por Salazar y De Morales (2012), quienes en su estudio realizado en el bofedal de Huaytire (3.000 - $4.500 \mathrm{msnm})$ observaron una temperatura media anual de $5{ }^{\circ} \mathrm{C}$, mientras que en el periodo sin hielo fue de $8,7^{\circ} \mathrm{C}$, con un mínimo de 7 ${ }^{\circ} \mathrm{C}$ y un máximo de $13,4^{\circ} \mathrm{C}$. Es probable que este tipo de valores sea considerado normal para este tipo de humedales altoandinos.

La correlación entre los iones bicarbonato y calcio es similar a la obtenida por Cooper et al. (2010), en sus estudios realizados en las turberas alpinas de los Andes (Región Jalca - Cajamarca), donde el calcio $\left(\mathrm{Ca}^{+2}\right)$ fue el catión más abundante en la mayoría de sus muestras, y su concentración se correlacionó positivamente con el $\mathrm{HCO}_{3}^{-}(\mathrm{r}=0,580)$. Estos iones conforman el bicarbonato de calcio o magnesio, presente en las rocas calizas de las formaciones Jumasha y Casapalca. La correlación entre el pH y la $\mathrm{CE}(\mathrm{r}=0,487)$ fue similar a lo obtenido por Vidal et al. (2011), en su estudio realizado en el Complejo de humedales El Yali (Chile) $(r=0,57)$. Esto se debe probablemente a que a medida que la concentración de sus iones provenientes del carbonato aumenta en la temporada seca, las medidas de $\mathrm{pH}$ tienden a incrementarse por la presencia de especies químicas de carbonato en el agua, adquiriendo un carácter ligeramente alcalino. Por otra parte, según Vitt (2006), en su estudio sobre las turberas boreales, el aumento de flujo de agua estacional podría generar una mayor entrada de nutrientes de nitrógeno y fósforo, que son independientes de los cationes y aniones básicos (minerales), pero que ambos podrían influenciar en la gradiente del $\mathrm{pH}$.

\section{Conclusiones}

En el presente trabajo se realizó la caracterización hidroquímica y su variabilidad espacio temporal de 
las aguas subterráneas en los bofedales altoandinos en la RPNYC, sector Moyobamba, sustentada en las mediciones de las propiedades fisicoquímicas de $\mathrm{pH}$, temperatura y CE, así como en el análisis de los constituyentes mayoritarios.

El tipo de agua dominante en las aguas subterráneas del bofedal de Moyobamba fue la bicarbonatada cálcica $\left(\mathrm{HCO}_{3}{ }^{-}-\mathrm{Ca}^{+2}\right)$ en el $95 \%$ de los pozos y la bicarbonatada magnésica $\left(\mathrm{HCO}_{3}{ }^{-}-\mathrm{Mg}^{+2}\right)$ en el 5\% restante, influenciadas por las características geológicas del bofedal.

$\mathrm{El}$ aumento de los iones bicarbonato $\left(\mathrm{HCO}_{3}{ }^{-}\right.$ ), calcio $\left(\mathrm{Ca}^{+2}\right)$, magnesio $\left(\mathrm{Mg}^{+2}\right)$ y sodio $\left(\mathrm{Na}^{+}\right)$, a medida que transcurría la temporada seca y su disminución en comparación con el mes de febrero, corresponde a la influencia que tiene la época del año sobre la hidroquímica del bofedal.

La conductividad eléctrica en el bofedal alcanzó sus valores más altos en la temporada seca (mayo a julio) y los más bajos en el mes de febrero. Por otra parte, presentó un carácter ligeramente ácido ( $\mathrm{pH} \cong 6,6)$. Esto probablemente se deba al proceso de descomposición que realiza la turba, que tiende a liberar sustancias con carácter ácido. Por último, la temperatura promedio del agua fue de $8,1^{\circ} \mathrm{C}$, considerado como un valor normal para estos bofedales altoandinos.

Se encontró una correlación positiva media, entre la concentración de los iones bicarbonato $\left(\mathrm{HCO}_{3}{ }^{-}\right)$ y calcio $\left(\mathrm{Ca}^{+2}\right)$, y entre el bicarbonato $\left(\mathrm{HCO}_{3}{ }^{-}\right)$y el magnesio $\left(\mathrm{Mg}^{+2}\right)$. Las aguas que abastecen al bofedal presentan carbonatos (calcita y dolomita) que provienen de la formación Jumasha y Casapalca. Además se encontró una correlación positiva débil entre el $\mathrm{pH}$ y la $\mathrm{CE}$, que corresponde al carácter ligeramente alcalino que se genera, por la presencia de carbonatos.

\section{Agradecimientos}

Se agradece el apoyo otorgado para la realización del trabajo de investigación, a través del convenio suscrito por la Universidad Católica Sedes Sapientiae, Patronato de la Reserva Paisajística Nor-Yauyos Cochas y la institución científica CORBIDI.

\section{Literatura citada}

American Psychological Association, American Water Works Association, Water Pollution Control Federation.

1992. Métodos normalizados para el análisis de aguas potables y residuales. Díaz de Santos. Madrid, España. $1830 \mathrm{p}$.

Belyea, L. R., \& Malmer, N.,

2004. Carbon sequestration in peatland: patterns and mechanisms of response to climate change. Global Change Biology,10:1043-1052

Cepeda Pizarro, J.

2013. Variabilidad temporal de algunos parámetros físicos e hidroquímicos de un prado húmedo alto-andino del nortecentro de Chile. IDESIA, 31(2), 89-97.

Cepeda Pizarro, J. \& Armijo León, A. A.

2014. Dinámica fluviométrica y limnológica de un cuerpo superficial de agua asociado a un prado húmedo de los Andes del norte-centro de Chile. IDESIA, 32(2):11-19.

Charman, D. J.

2009. Peat and Peatlands. Earth Systems and Environmental Sciences, 541-548.

Cooper, D., Wolf, E., Colson, C., Vering, W., Granda, A. \& Meyer, M.

2010. Alpine Peatlands of the Andes. Arctic, Antarctic, and Alpine Research, 42 (1):19-33.

Durán, V., García, A., Robledo, P.

2009. Propuesta de clasificación genético-geológica de humedales. Aplicación a los humedales españoles incluidos en el Convenio de Ramsar. Boletín Geológico y Minero, 120 (3): 335-346.
Flusche, M. A., Seltzer, G., Rodbell, D., Siegel, D., Samson, S. 2005. Constraining water sources and hydrologic processes from the isotopic analysis of water and disolved strontium, Lake Junín, Perú. Journal of Hydrology, 312: 1-13.

Ginocchio, R., Hepp, J., Bustamante, E., Silva, Y., De la Fuente, L., Francois, J., De la Harpe, J., Urrestarazu, P., Anic, V.; Montenegro, G. 2008. Importancia de la calidad del agua sobre la abundancia y la diversidad vegetal en vegas altoandinas del Santuario Natural Yerba Loca en los Andes de Chile centro-norte. Revista Chilena de Historia Natural, 81: 469-488.

Holden, J., Chapman, P.; Labadz, J.

2004. Artificial drainage of peatlands: hydrological and hydrochemical process and wetland restoration. Progress in Physical Geography, 28(1): 95-123.

Korswagen Eguren, S.

2016. Análisis espacial del hábitat de la vicuña en la comunidad campesina de Tanta, en la Reserva Paisajística Nor Yauyos Cochas. Espacio y Desarrollo, (28): 103-128.

Lecomte, K., García, G., Fórmica, S.; Depetris, P. 2011. Hidroquímica de ríos de montaña (Sierras de Córdoba, Argentina): Elementos mayoritarios disueltos. Latin American Journal of Sedimentology and Basin Analysis, 18 (1): 43-62.

Maldonado Fonkén, M.

2015. An introduction to the bofedales of the Peruvian High Andes. Mires and Peat, 15: 1-13.

Megard, f., Caldas, J., Paredes J.; De la Cruz, N. 1996. Geología de los cuadrángulos de Tarma, La Oroya y Yauyos. INGEMMET, Boletín Nº 69. INGEMMET. Lima, Perú. 279 p. 
Ministerio del Ambiente.

2011. Inventario y evaluación del Patrimonio Natural en la Reserva Paisajística Nor Yauyos Cochas. MINAM.Lima, Perú. 289 p.

Oyague Passuni, E.; Maldonado Fonkén, M.

2015. Relationships between aquatic invertebrates, water quality and vegetation in an Andean peatland system. Mires and Peat, 15: 1-21.

Romanelli, A., Quiroz Londoño, O., Massone, H., Martínez,

D. \& Bocanegra, E.

2010. El agua subterránea en el funcionamiento hidrológico de los humedales del Sudeste Bonaerense, Provincia de Buenos Aires, Argentina. Geológico y Minero, 121(4):386.

Ruthsatz Trier, B.

2012. Vegetación y ecología de los bofedales altoandinos de Bolivia. Phytocoenologia, 42 (3): 133 - 179.

Salazar Torres, G. \& De Morales Huszar, V.

2012. Microalgae community of the Huaytire wetland, an Andean high-altitude wetland in Peru. Acta Limnologica Brasiliensia, 24 (3): 285-292.

Salvador, F., Monerris, J. \& Rochefort, L.

2015. Peatlands of the Peruvian Puna ecoregion:types, characteristics and disturbance. Mires and Peat, 15: 1-17.
Sánchez Sánchez, J., Álvarez Legorreta, T., Pacheco Ávila, J., González Herrera, R. \& Carrillo Bribiezca, L.

2015. Caracterización hidrogeoquímica de las aguas subterráneas del sur del Estado de Quintana Roo, México. Revista mexicana de ciencias geológicas, 32 (1): 62-76.

Squeo, F., Warner, B., Aravena, R. \& Espinoza, D.

2006. Bofedales: high altitude peatlands of the central Andes. Revista Chilena de Historia Natural, 245-255.

Vásquez, C., Ariza, A.; Pinilla, G.

2006. Descripción del estado trófico de diez humedales del altiplano cundiboyacense. UNIVERSITAS SCIENTIARUM, 11 (2): 61-75.

Vidal Abarca R., Suarez, L., Figueroa, R., Enríquez, M., García, V., Domínguez, C.; Arce, I.

2011. Caracterización hidroquímica del complejo de humedales El Yali, Chile. Limnetica, 30(1), 43-58.

Vitt, D. H.

2006. Functional Characteristics and Indicators of Boreal Peatlands. In: Wieder, R.K. \& Vitt, D.H. (eds.) Boreal Peatland Ecosystems. Springer-Verlag.Berlin, Heidelberg. Germany. Pp 9-24. 
